\title{
Impact of Large Scale Wind Power Integration on Small Signal Stability
}

\author{
Ângelo Mendonça, and J. A. Peças Lopes, Senior Member, IEEE
}

\begin{abstract}
This paper deals with the problem of large wind power integration and its potential impact on systems small signal stability. Factors like grid configuration, load and wind power integration are considered. An automatic algorithm is used to search the space formed by such parameters and generate a representative set of possible operation conditions. The oscillation modes are then calculated for each operation point. Some of these points of operation are subjected to a deeper analysis and the movement of oscillation modes on the complex plane is evaluated. Finally, non-linear time domain simulations are presented.
\end{abstract}

Index Terms--Small signal stability, wind power

\section{INTRODUCTION}

$\mathrm{W}$ IND power has achieved nowadays a high level of integration in many power systems. This fact is leading to significant changes in power systems operation that have been studied by grid operators, wind turbine manufacturers and scientific community. Power system dynamics and transient stability has received considerable attention, namely concerning the performance of wind turbines, their response to short circuits, and the impact of such large disturbances on the grids. In earlier years small signal stability problems received considerable less attention, but as wind power integration increased some concern about possible negative effects has arisen. The most frequent cause of this kind of instability is the lack of damping of the so called electromechanical modes, which are related with low frequency $(0.1-2 \mathrm{~Hz})$ power oscillations that occur in machines rotor, essentially due to the settings of generator controllers and the efforts to transmit bulk power through weak transmission lines [1].

Because of its characteristics it is possible that wind power, under some circumstances, may contribute to reduce system damping. In fact, wind power resources are generally located far from large centers of consumption, while generators that are not to be commited, typically thermal units, are located closer to major loads. This may involve transmitting energy for long distances through congested lines and perhaps a significant change of generation profile and typical power flows, which may have significant impact on small signal stability.

This work was supported by the Fundação para a Ciência e a Tecnologia (FCT) of Portugal Grant SFRH/BD/18469/2004.

Ângelo Mendonça is with the Power Systems unit of INESC Porto, Porto, Portugal (e-mail: angelo.mendonca@ inescporto.pt).

J. A. Peças Lopes is with the Power Systems unit of INESC Porto and with the Department of Electrical and Computer Engineering, Porto University, Porto, Portugal (e-mail: jpl@fe.up.pt).
A first approach to this problem is reported in [2]. It is concluded that wind turbines themselves do not lead to power system oscillations. Moreover, it is shown that in the squirrel cage induction generator as well as in variable speed wind turbines, rotor oscillations are much better damped. Later studies carried out for the Nordic Grid have shown that under some circumstances the integration of high levels of wind power production may lead to a reduction of damping [3]. The reasons for this are however not yet well investigated.

The objective of this research is to identify how large scale integration of wind power affects modes of oscillation. The adopted approach consisted in considering a test system and generating a representative data set of different system operating conditions like network configuration, load level, wind power integration levels, wind power dispersion, and unit commitment of conventional units. The data set was first analyzed to identify the driving forces that influence damping. Then some operation points were selected for a more detailed study, using modal analysis, to track the evolution of the oscillation modes when wind power is increased. This approach allows studying all possible operating scenarios, avoiding in that way a biased analysis.

The operating scenarios were automatically generated using a Matlab program developed for that purpose that also allows carrying out modal analysis. Nonlinear time domain simulations were carried out using PSS/E software package. A test system with three areas was used throughout this research.

The following section describes the system modeling. In section III the test system is described and some results are presented. Finally, conclusions are outlined in section IV.

\section{ADOPTED APPROACH}

\section{A. System Modeling}

The most adequate tool to perform small signal stability studies is modal analysis [4]. This assumes a linear model of the power system, in a state space form. The canonical description is obtained from the analytical linearization of the set of differential equations that define the state model of the power system. The eigenvalues of the state matrix are computed, from which are then calculated the damping conditions associated to each oscillation mode. For detailed analysis, mode shapes, participations factors and residues are also used.

Synchronous generators are modeled using a six-order model, with magnetic saturation neglected, where voltage regulators are included and modeled with an IEEE type 1 model [5]. Wind power production is represented by variable 
speed wind turbines, using the simplified model proposed in [2] that consists of active power and voltage regulation loops, which control active and reactive power injected.

\section{B. Data Set Generation}

A simple structured algorithm that searches the space formed by the parameters of interest (network configuration, load and wind power) was used in this research to generate a set of operation points. The developed algorithm is based on the work presented in [6] although Monte Carlo sampling was not implemented. Since the system used is small, the computational time required to generate all possible combinations with a small resolution is acceptable and a simple searching algorithm was found adequate. If larger systems are considered a structured Monte Carlo sampling algorithm may then be applied. Because no random numbers are used, the simple algorithm used, allows duplicating studies when necessary.

The automatic procedure developed to generate the data set (DS) consists of the following steps:

a) The DS operating range is defined and partitioned according to the range and resolution assigned.

b) For each configuration and load scenario, all possible combinations of connecting the available wind power are considered and assigned.

c) The necessary conventional production is dispatched distributing the difference between load and total wind power integration. All possible scheduling combinations are considered.

d) For each selected dispatch solution, the steady-state operating conditions are obtained through a power flow calculation. Before accepting the results the feasibility of the power flow solution is checked regarding the operational restrictions.

e) The linear model of the power system is then obtained for each accepted steady-state solution. From these results a damping index may be defined. In this research the poorest damped mode in considered.

Although only three parameters are defined, due to the dispersion introduced by the DS generation algorithm, an operation point belonging to DS must be characterized by network configuration, total load, wind production level and location and conventional production connected at each bus.

\section{NUMERICAL RESULTS AND DISCUSSION}

\section{A. Test System}

The system presented in Fig. 1. is used in this research to assess the impact of wind power on small signal stability. The system is derived from the well known two area test system, widely used for stability studies [5]. A third area was created, adding to bus 8 two generators similar to G3 and G4, a load and a capacitor, so that a second inter area mode arises. Wind power is connected at buses 6,8 and 10 . The values of generation, load and capacitors are presented in Appendix I. All the other data maintain the original values presented in [5].

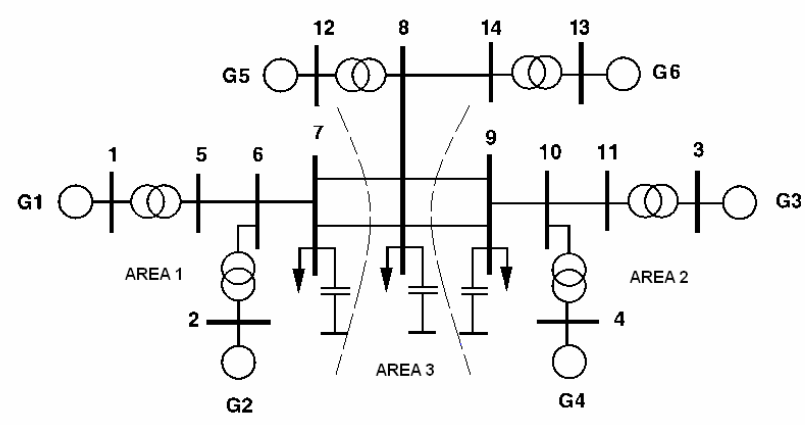

Fig. 1. Test system with three areas

Note that the generators do not represent a single machine but a group of strongly coupled generators. In the original data these are units of $900 \mathrm{MVA}$, and for this study it was assumed the existence of 5 machines of 180 MVA each. The transformers connecting generators to the grid are adjusted accordingly. Wind turbines are the $2 \mathrm{MW}$ machines described in [2] and are considered to operate at unit power factor. Reactive power is supplied by capacitors connected to the same bus such that injected reactive power is $20 \%$ of active power.

\section{B. Studied Scenarios}

The studied scenarios were automatically generated using the algorithm already described. The operating ranges and resolution for the test system were the following:

Configuration: normal and first order contingencies; Load: varying from 2000 to $3600 \mathrm{MW}$ in $800 \mathrm{MW}$ steps;

Wind: varying from 0 to $1200 \mathrm{MW}$ in $300 \mathrm{MW}$ steps.

Only contingencies of interconnection lines were considered. System load was modified scaling the values presented in appendix, so the proportion of loads between buses and between active and reactive power are kept constant. Capacitors were defined to compensate reactive power consumption.

For reference, the scenario without wind power may be considered as the base scenario. Generation, loads and capacitors values for this scenario are presented in Appendix I. All areas have a total production of $1200 \mathrm{MW}$ and approximately $200 \mathrm{MW}$ are exported from area 1 to area 2, through area 3.

\section{Data Set Analysis}

The generated data set provides interesting results but its analysis is a difficult task due to the amount of data and its multidimensional nature. Fig. 2 shows the single dimension projection of the damping, sorted in decreasing order, the corresponding configuration, the total load and total wind power production. It is possible to observe in the first graphic of Fig. 2 that several kinds of scenarios have been generated: well damped, insufficiently damped and unstable. By simple inspection is possible to conclude that large wind power integration can have a positive or negative impact under almost every operating condition. It is therefore important to determine which factors influence damping. 

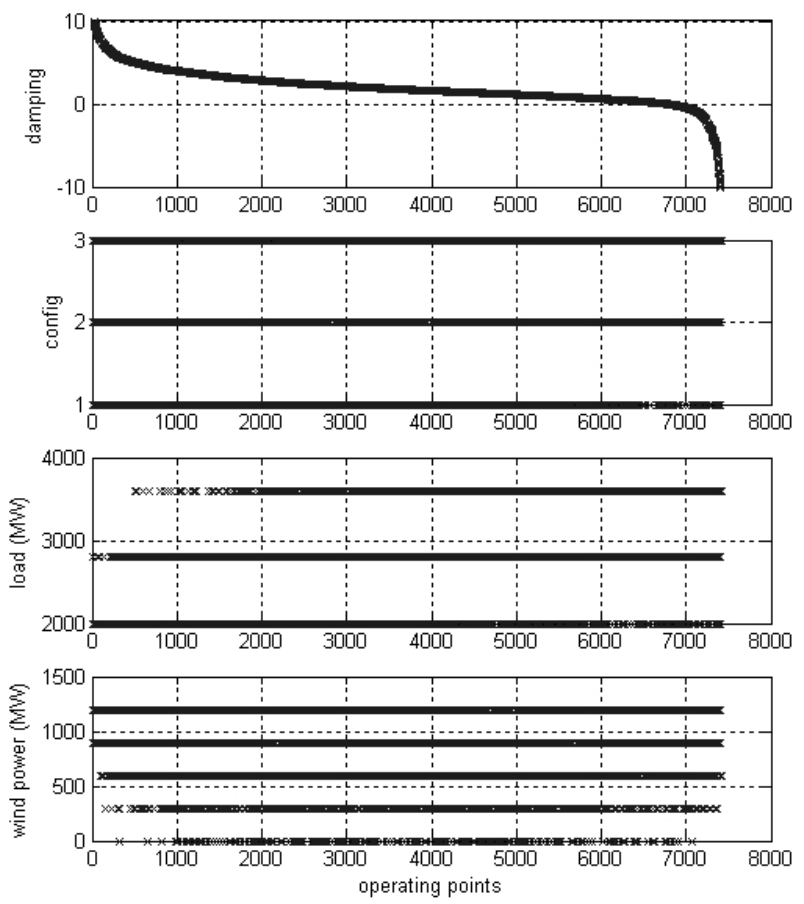

Fig. 2. Operation points characteristics

Fig. 2 also shows the factors that characterize each operating point. The second graphic characterizes the configuration, where 1 means the normal configuration with all branches in service, 2 means that one line connecting bus 7 to bus 8 is out of service and 3 means that one line connecting bus 8 to bus 9 is out of service. The third graphic shows total load value and fourth graphic shows wind power production. It is possible to conclude that none of these factors explain the variation of damping since, for the same configuration, the same load and the same wind power penetration, there are well damped modes, insufficiently damped modes and unstable modes. For that reason these are not relevant factors when evaluating impact of wind power integration. Note that this does not mean that network configuration and load level do not influence damping but just that knowing all these factors is not enough to determine the damping conditions. For example, for the same load and unit commitment, a scenario with a branch out of service will be, of course, worse damped.

The damping variation must be explained by the dispersion of the production. Fig. 3 shows the operation points that consider only normal configuration, total load of $3600 \mathrm{MW}$ and wind power production of $1200 \mathrm{MW}$. For reference, operation points without wind power are shown with a different marker. It is shown the damping of the generated operation points sorted in decreasing order and the production of conventional units in each area. It is possible to observe that, in general, removing conventional production from area 3 leads to a reduction of damping. In general, increasing production of areas 1 and 2 leads to a reduction of damping. Results also confirmed that replacing conventional production by wind power within the same area increases damping.
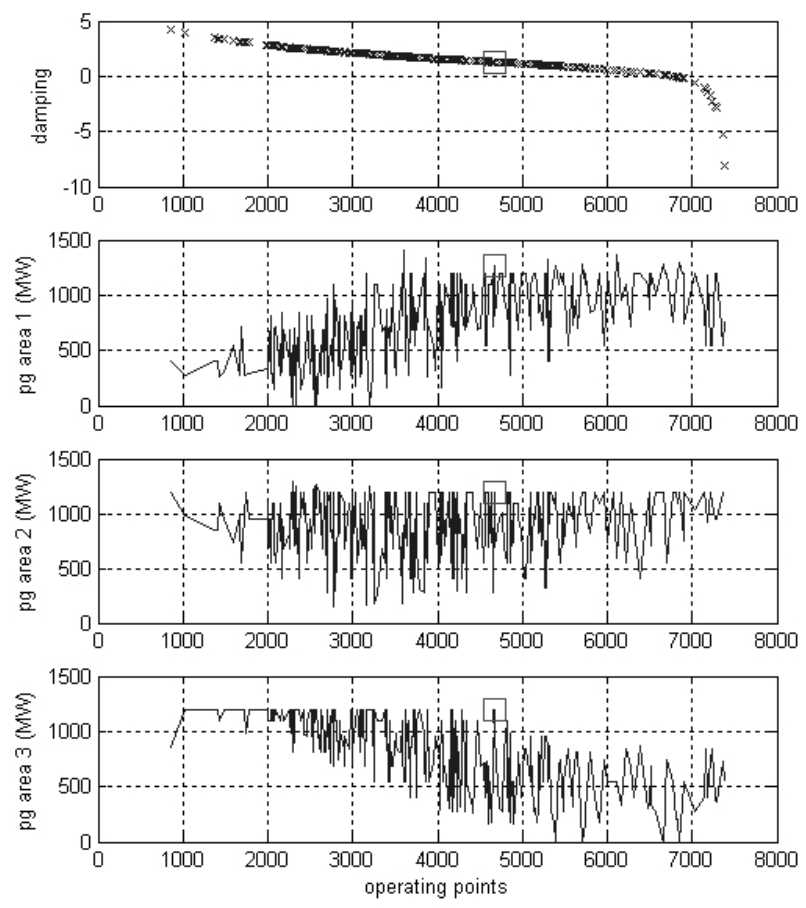

Fig. 3. Operation points characteristics

Changes in damping are an overall result of many factors that change in a network when wind power is increased. This overall result depends also of system configuration, which may explain some discrepancies between studies [2][3]. For a larger system, with a high number of inter area modes such analysis may even be more complex.

\section{Modal Analysis}

A more detailed analysis of three cases of wind power integration is presented in this section: a) conventional production located near loads is replaced by wind power mainly located far from loads b) wind power replaces conventional production within the same area and c) wind power used only to supply load increase. These studies were conducted selecting an initial scenario, with small wind power integration, that is increased gradually. The initial scenario corresponds to the normal configuration, a total load of $3600 \mathrm{MW}$ and $400 \mathrm{MW}$ of wind power connected do bus 8 that, was compensated reducing the production of generator 4 . This scenario has five modes of oscillation: one intra area mode for each area, an inter area mode corresponding to the oscillation between area 3 and areas 1 and 2, and a low damped mode corresponding to the oscillation between area 1 and area 2. Intra area modes are well damped and not relevant in this research. The poorest damped mode is the one that presents the lowest frequency and corresponds to the oscillation of generators in extreme areas against each other. The other inter area mode corresponds to the oscillation of generators in area 3 against the others.

The first study was conducted reducing gradually conventional production in area 3 replacing it by wind power con- 
nected to bus 10, in area 2. Fig. 4 shows the movement of the oscillation modes on the complex plane, with the squares representing the initial conditions and circles the final operating conditions. An arrow also indicates the movement direction.

It is clear that damping of the lowest frequency mode decreases, while damping of the other inter area mode increases significantly. The damping reduction can be explained by the increase of load in line 9-10. Another important effect is the modification of the mode shapes. Because power of G6 is gradually reduced until the generator is switch off, the intra area mode of area 3 no longer exists in the final scenario. For the same reason the inter area mode of higher frequency evolves to a local mode of G5.

The second study was conducted increasing gradually the wind power in area 1 , connected to bus 6 , and reducing by the same amount the conventional production within the same area. The movement of the oscillation modes in the complex plane is shown in the Fig. 5, where it is possible to verify that a small increase of damping occurs for both inter area modes.

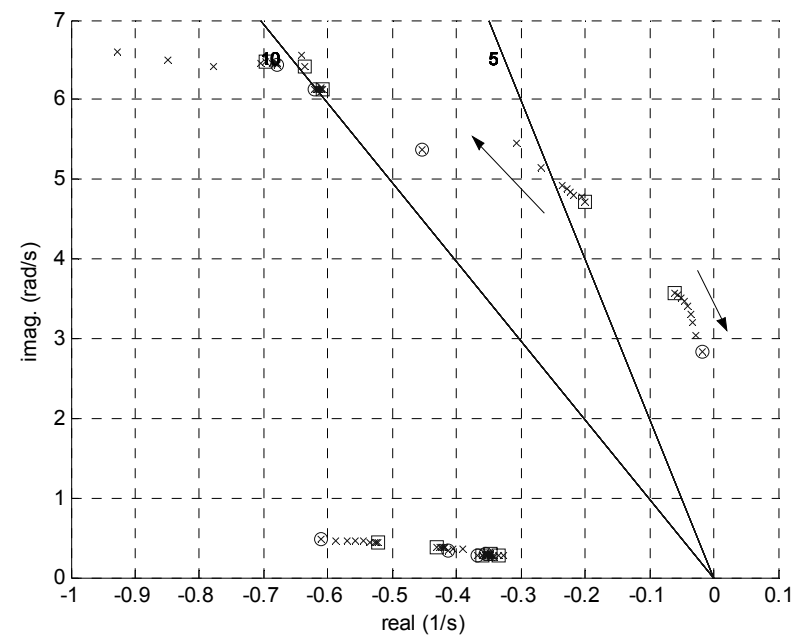

Fig. 4. Pole map: movement of the oscillation modes when wind power increases in areas 2 and conventional is reduced in area 3

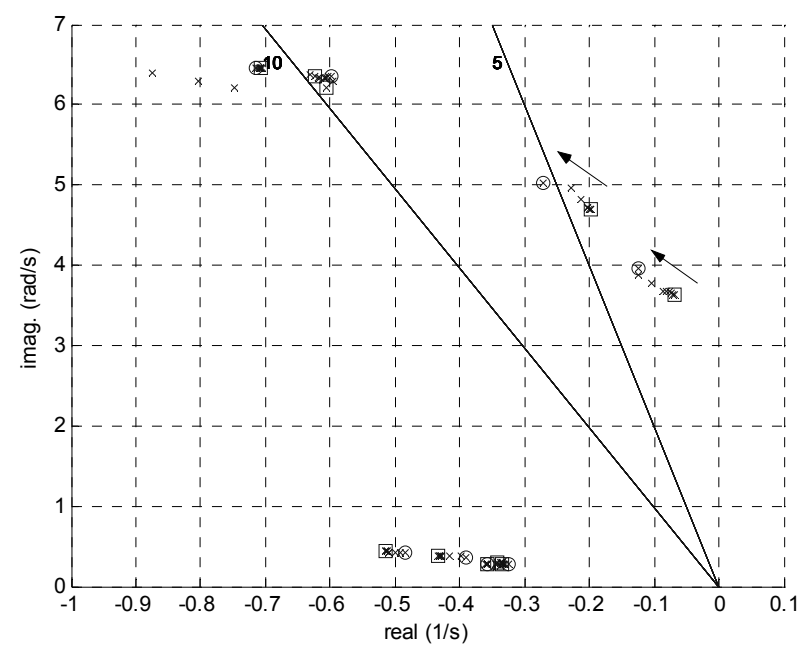

Fig. 5. Pole map: movement of the oscillation modes when wind power increases replacing conventional production in area 1

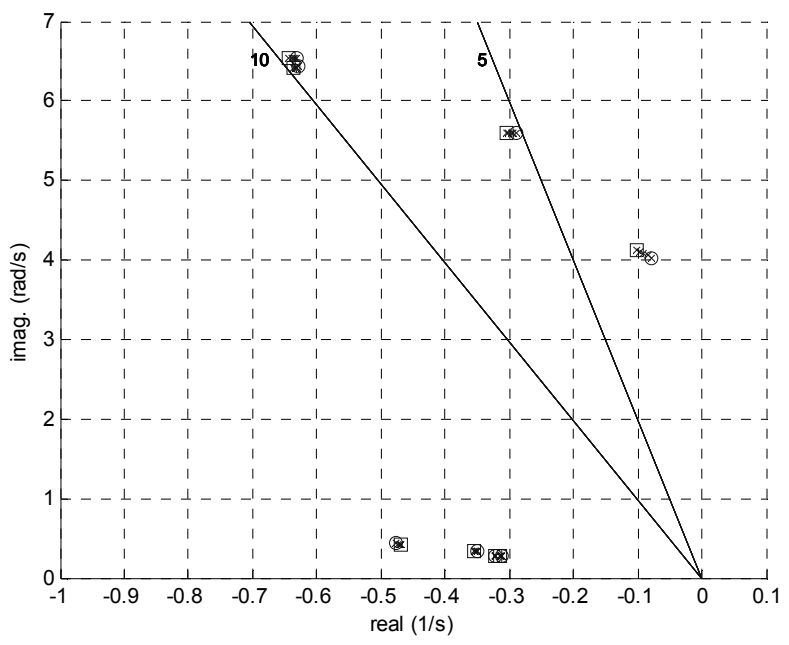

Fig. 6. Pole map: movement of the oscillation modes when wind power and load increases in all areas

The final scenario for the third scenario consists of gradual load increase assuming that the new consumptions are supplied by wind production connected to the same areas (buses 6,8 and 10). Conventional generation is therefore not switched off. Fig. 6 shows the movement of the oscillation modes on the complex plane.

When wind power integration follows load increase, damping of both inter area modes decreases slightly, most likely because load of lines between production and consumption also increases. Other modes are not significantly affected and mode shapes structure does not change.

\section{E. Time Domain Analysis}

Since power systems behavior is highly non linear, time domain simulations play an important role in stability studies. It is therefore useful to validate results from modal analyzes and to observe in a clear way the behavior of generators. These simulations also allow studying the way oscillation modes are excited and observable in system response. When a disturbance is applied several modes are excited and some may not be observable, creating difficulties to analyze the results. For the test used this task is someway simplified because few generators participate in the oscillation modes.

Fig. 7 and Fig. 8 show the speed deviation of a synchronous machine from each area after a short circuit in the bus 10 for base scenario and final scenario of case 1, illustrated in Fig.4. The figures were obtained from simulations carried out using PSS/E software package and modified to improve clearness. Comparing both figures it can be confirmed that increasing wind power integration according to pattern 1 damping is reduced and changes in the mode shape structure can be observed. It is also interesting to note that, although generators of area 2 are closer to the disturbance, their participation in the oscillations is less relevant then generator 1 .

If damping of the new modes is not acceptable, measures to increase damping, like power system stabilizers, are recommended to be installed. The changes produced by large scale 
integration of wind power may have some impact on the way PSS are tuned since the structure of power system oscillations, under some conditions, suffers large modifications.

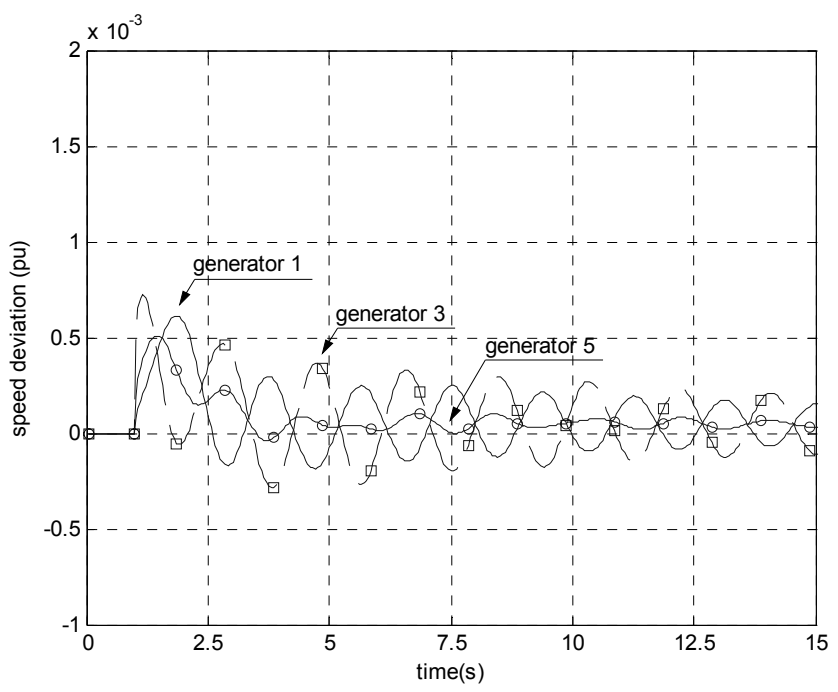

Fig. 7. Speed deviation after a short circuit in bus 10: base scenario

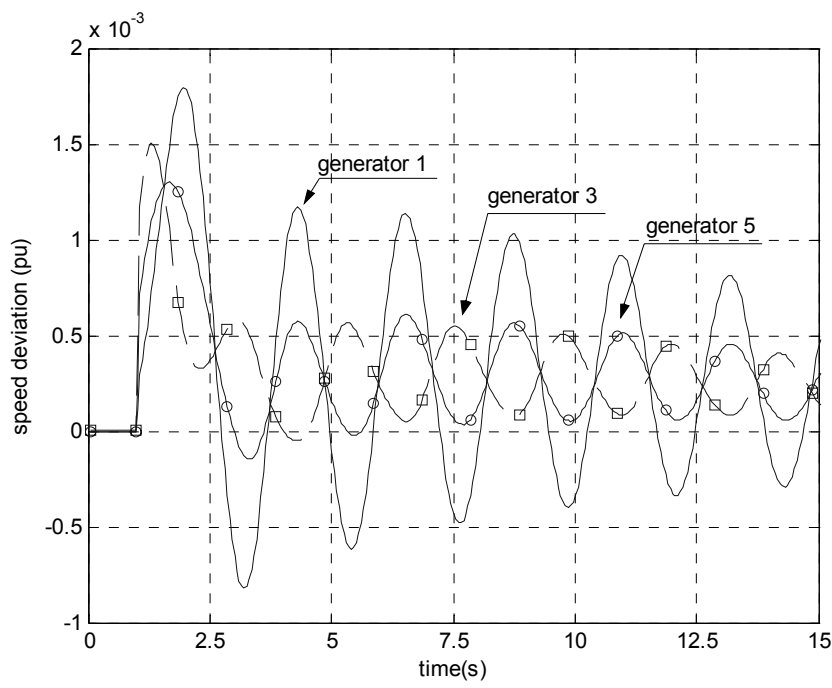

Fig. 8. Speed deviation after a short circuit in bus 10: final scenario of case 1

\section{CONCLUSIONS}

The research described here aims to identify the impacts on small signal stability of increasing the integration of wind power. This problem is not sufficiently studied, which may lead system operator to fear large scale integration of wind power in system with low damped power oscillation.

Although wind generation is, in general, not worsening damping regarding inter area oscillations, the changes in the operational structures of the systems may lead to considerable reduction on the damping of these modes.

Several patterns of increasing wind power and load were considered in this study and for each scenario the damping conditions were computed. It was then observed that damping is reduced when wind power increases and contributes to con- gest weak interconnection lines. Inter area modes are the most sensitive ones. Measures to mitigate these impacts, like power system stabilizers, may then need to be installed and properly tuned to ensure an adequate operation.

Further research using cluster analysis is being pursued for better understanding the problem and to identify the main factors that influence damping.

\section{APPENDIX}

This appendix presents the data of the test system used in this research, modified from the original data presented in [5].

TABLE I

TEST SYSTEM DATA

\begin{tabular}{|c|c|c|c|}
\hline Bus & $\begin{array}{c}\text { Load } \\
\text { (MW/Mvar) }\end{array}$ & $\begin{array}{c}\text { Generation } \\
\text { (MW) }\end{array}$ & $\begin{array}{c}\text { Capacitor } \\
\text { (Mvar) }\end{array}$ \\
\hline 1 & & 600 & \\
\hline 2 & & 600 & \\
\hline 3 & & 600 & \\
\hline 4 & & 600 & \\
\hline 7 & $1000+\mathrm{j} 100$ & & 100 \\
\hline 8 & $1200+\mathrm{j} 100$ & & 100 \\
\hline 9 & $1400+\mathrm{j} 100$ & & 100 \\
\hline 12 & & 600 & \\
\hline 13 & & 600 & \\
\hline
\end{tabular}

\section{REFERENCES}

[1] M. A. Pai, D. P. Sen Gupta and K. R. Padiyar, Small Signal Analysis of Power Systems, Harrow: Alpha Science International, 2004.

[2] J. G. Slootweg, "Wind Power: Modelling and Impact on Power System Dynamics", PhD thesis, Delft University of Technology, 2003.

[3] Espen Hagstrøm, Ian Norheim and Kjetil Uhlen, "Large Scale Wind Power Integration in Norway and Effect on Damping in the Nordic Grid", in Proc. Nordic Wind Power Conference Conf.., 1-2 March, 2004 Chalmers University of Technology.

[4] L. Rouco, "Eigenvalue-based Methods for Analysis and Control of Power System Oscillations", IEE Colloquium on "Power Dynamics Stabilization", University of Warwick, Coventry (England), February 1998.

[5] P. Kundur, Power System Stability and Control, New York: McGrawHill, 1994.

[6] H. Vasconcelos, J.N. Fidalgo, J.A.P. Lopes, "A General Approach for Security Monitoring and Preventive Control of Networks with Large Wind Power Production", Proceedings of PSCC02 - 14th Power Systems Computation Conference, June 2002. 